\section{D.R. Adam}

Technion -

Israel Institute of Technology

Dept. of Biomedical Engineering

Haifa, Israel

The fast growing areas of industrial research and development, which are highly motivated by the requirements of up-to-date clinical services and support, are image guided therapy/surgery and telemedicine (either from the operating room to the office of an expert, or remotely to his location in a foreign country, or to an archiving and storage headquarters). These areas of growth are stimulating the development of new methods of Signal Processing and Image Processing, specifically towards more automatic analysis, segmentation and classification.

Signal processing algorithms of some of the physiological signals have been developed and matured to such an extent that the required pattern (e.g. the QRS complex in the ECG signal) can be identified withextremely high accuracy and reliability. It is therefore understandable that efforts are currently invested in studying minute changes in such waveforms, and developing methods of identifying and tracking the minuscule changes over time. The choice of sensitive indices, and the study of their correlation with pathophysiological or clinical events - have been the focus of recent reports. The study of traditional vs. transformed ECG-based indices, as a tool for identifying occluded arteries in patients with myocardial ischemia, induced by prolonged Percutaneous

\title{
Synopsis
}

\section{Signal and Image Processing}

Transluminal Coronary Angioplasty (PTCA), was reported this year by J. Garcia et al., [1]. While their study is based on specific indices, most of them clinically accepted ones, a different approach was taken by R. Vetter et al., [2]. They chose a more general approach, requiring very little a-priory information: the method of Blind Source Separation. This method is an unsupervised learning scheme, which was used [2] for studying changes of activity within the autonomic nervous system.

The report of Garcia et al., attempts to correlate spatial properties of ischemic changes (caused purposely under controlled conditions of a PTCA protocol), withchanges ofECGindices. While commercial arrhythmia monitoring systemsusuallyidentifyECGcomplexes, or major parts of these complexes, and classify them according to predefined clinical classes, and ECG analyzers can follow changes in a specific section(e.g. ST) over time, Garcia et al. attempt to correlate surface ECG indices with location of occlusion of one of the coronary arteries. Such an effort has been attempted before, with limited success, since occlusion of a coronary artery at a specific location may cause different ischemic patterns of the ECG in different patients (caused by the patient's chestmorphology, location of the heart, collateral blood flow etc.). Garcia et al. have selected a set of parameters measured from the standard 9 electrode ECG time recordings - voltages measured at specific times during the cardiac cycle, time durations of certain waveforms, etc. They have measured the changes of these parameters during transient occlusions, made at different locations in the coronary artery tree. They have also selected several global indices, which were calculated by performing the Karhunen-Loeve Transform (an optimal orthonormal transformation) over specific sections of the ECG waveform. The advantage of this approach is its ability to capture the important information and ignore the small details (by selecting the largest Eigenvalues of the covariance matrix). Garcia et al. have used multivariate discriminant analysis in order to select the set of indices that best correlate withthe location of the (deliberately caused) transient ischemia. It is not surprising to learn that the global indices produced better discrimination than the local indices, among the locations of the occlusion. It is also not surprising to learn that a combination of global and local indices produced even better results (correct classification of $90 \%$ of the patients). This study is a good example of the power of good statistical analysis, but also of its limitations: a 9 lead ECG can provide only limited information about underlying physiological processes. There is need for either more electrodes (better spatial resolution), invasive endocardial measurements or other clinical information. 
The approach taken by Vetter et al. [2] requires very little information on the data (e.g. no need to measure the waveform or amplitude at specific times) but it does require assuming a (linear) model of the system generating the data, and independence of the different sources affecting it. In this report the authors describe their study of the baroreflex control loop: They measured two signals - the Heart Rate and the arterial blood pressure. By assuming that the signal sources affecting these measurements, the sympathetic and parasympathetic nervous activities, are independent of each other-Vetter et al. try to estimate from the above measurements the nerve activity of each source (with emphasis on the sympathetic nervous activity). The same method can be applied to many other physiological signals - but its usefulness depends on whether a model may be well matched to the biological system studied. This is not always the case. But when the model describes accurately the system (under some specific conditions of the study) - the technique of blind source separation is an extremely powerful tool for separating and segmenting various sources so that their combination is measured at the output of the model/system. Some reports claim that up to seven independent sources can be separated by this technique. One of the computational issues related to this technique is the need to adjust (blindly) the demixing parameters of the reconstruction model. Anerror function should be defined (e.g. minimization of an appropriate contrast function based on maximum likelihood, minimum entropy or other measure), to provide an update rule for the demixing parameters. Though there is no guarantee that the updating rule will cause convergence, there are methods that impose this convergence.

The first two reports cited above dealt with one-dimensional signals, but the techniques they describe can be extended to 2D images. The report of McInerney et al., [3], as well as the other reports described later, describe techniques applied to 2D images. When the problem of separation or segmentation of different signals, of various waveforms (or generated by various underlying mechanisms), is extended to $2 \mathrm{D}$ - segmentation lines or even surfaces must be used. Medical images usually describe cross sections of organs, tissues etc., and one of the first requirements is to segment the image into regions belonging to the different organs (e.g. kidney, myocardial tissue, blood pool etc.). McInerney et al. have extended the method which applies deformable models to an adaptive one, which they term topology adaptive deformable surface. The method is an extension of the very popular and useful class of deformable models, deformable contours, termed 'snakes'. Snakes have become a popular tool, since this tool has been easy to implement, its computational complexity is low, thus it can be applied in real-time, and the results it produces are good. It is an optimization procedure that takes into account 'internal forces' and 'external forces', which are terms describing elements of the cost function: the 'internal forces' control the high spatial frequencies of the 'snake' enabling it to fit better (or less) protrusions and concavities; the 'external forces' are 'forces' generated by the image, whichexpand/inflate the 'snake' or attract it to edges. This optimization procedure is not a global one, but in most cases it does not collapse into a local minimum, mainly because in most cases it is initiated by a different procedure so that it actually functions only in close proximity to the expected, real contour. McInerney et al. have taken this procedure further. They have earlier reported on an adaptive ‘snake' method, which exploits a mechanism that induces an efficient re-parameterization mechanism (termed ACID). Thus the 'snake' in their case can adapt to different geometries within the image - and modify itstopology to describechanging structure (e.g. from that of a section of blood vessel - to a full arterial tree). In the present report [3] they extend the method to segmentation of volumes. The topology adaptive deformable surfaces utilize an extension of the ACID framework, so that various volumes can now be segmented, with the deformable surfaces being dynamically adapted to that of the target object. There is no need to initialize the procedure by drawing the surface close to the target surface. The deformable surface can be initiated anywhere in the volume, and it is supposed to grow and move around, adapt itself to various 3D morphologies, until it fits the desired volume. The procedure is claimed to be nearly automatic, and nearly independent of initial conditions, yet interactive in the sense that the operator can control and manipulateit.

Once surface (or contour) segmentation is performed, one of the key clinical applications is to tie it with the properties of the tissue. Kyriacou et al. [4] have reported on their study of nonlinear elastic registration of $2 \mathrm{D}$ brain images with tumor pathology, using a biomechanical model. By using a finite-element mechanical model of the tissues (in the brain), they have been able to calculate the interaction between the different forces within the tissues, generated by the growth of a tumor, and to demonstrate the normal structure, before the tumor growth. This nonlinear elastic registration allows comparison of the 'back to normal' anatomy with atlases of organs (brain) of normal patients, thus enabling exact identification of each structure. Once each structure and tissue is identified, 
the 'normal' anatomy can be deformed back - to describe the anatomy with the tumor grown to its full size, with all details identified. This maneuver is extremely important for identifying crucial structures within the brain, to avoid critical damage during surgery. It also allows planning of surgery, including estimation of the anatomy after tumor removal, when new biomechanical balance must be achieved. The method Kyriacou et al. describe is a nonlinear optimization approach, by which the changes during tumor 'removal' or 'regrowth' are calculated. The deformation thus calculated, must take into account tissue properties, boundaries of brain structures (skull, ventricles etc.), thus the operation is global for the whole brain cross section. This limits the procedure to major anatomical changes, and does not allow studying small, localized details. Future work will probably extend this approach to volume calculations and will enable emphasis of local changes.

The advances in methods of image processing and analysis have provided the basis for extensive usage of images in nearly all clinical environments. Clinicians rely on images for diagnosis, treatment control etc. The original images, processed images and database images must be stored, transferred from location to location etc. Thus an extremely important issue is that of compression of images, specifically lossless compression. Munteanu et al. [5] describe an algorithm based on the wavelet transform, for compression of angiographic images. These images usually include large areas with no details, while the arteries imaged must be described in high spatial resolution and accuracy. Application of the wavelet transform is very appropriate, since it allows description of such high spatial frequencies at the specific locations, while using low resolution coefficients for the 'background'. One of the main advantages of this approach is its low complexity - and since the compression is lossless its implementation is computationally cheap yet it saves storage space and allows faster transfer of images over given communication channels.

Once the medical images have been processed, segmented, stored and retrieved, they have to be labeled, annotated, and integrated into medical information systems. These topics are covered by the papers of Mori et al., [6] and of Golland et al. [7]. Mori et al. describe a method of automated anatomical labeling of each bronchial branch. This procedure, or a comparable one, is important for implementing computer aided diagnostic systems of medical images. The authors have developed their method for 3D chest CT images. The 3D approach, though it seems more complex, actually allows better interpretation of the complex spatial relations between branches within the tree structure of the bronchus. The method depends of course on a knowledge base of the bronchial branch name, which holds this information as a set of rules for its anatomical labeling. It is essential for any such attempt of automatic anatomical (or functional) labeling to first develop the appropriate knowledge base and data base, which must be developed separately (usually demanding a major investment of time and effort). The method developed by Mori et al. requires definitions of evaluation values and cost functions, so that relations among branches could be identified and organized in the order best fitting the rules set by the knowledge base. The major future investment needed is the development of a large knowledge base, which should include a large variety of bronchial structures.
As described above - the next required step is to implement the annotated and labeled images within a medical information system - so that the images can be correlated with other images (either of the same subject - or of other subjects), can be merged with other information sources (images acquired by other modalities, functional images, clinical data, etc.), and all this wealth of information should be simply displayed. Golland et al. [7] describe such an effort. The system they have conceptually developed combines a novel technique for visualization (independent of the hardware of the imaging system - thus allowing cross-platform implementation), together with a method for performing annotations and cross-referencing of clinical and image data. The system allows integration of 3D surface models (generated by the system) with 2D images of cross sections and textual data. The main goal of this effort was to produce a user friendly system, which is very simple to operate, yet allows powerful operations of data integration and management, and meaningful display of 3D organs. This effort is a major step in integrating all the previous steps as described in this Synopsis so as to produce a clinically useful tool.

The papers reviewed in this synopsis are just a very small sample of the huge number of studies performed in recent years whose purpose has been to produce clinically useful information systems. It is important to note that although there are still numerous unsolved problems before completely automatic reliable (and inexpensive) systems are available - the efforts of recent years demonstrate that major achievements have been made, and basic systems are already available. 


\section{References:}

1. García J, Wagner G, Sörnmo L, Lander P, Laguna P. Identification of the Occluded Artery in Patients with Myocardial Ischemia Induced by Prolonged Percutaneous Transluminal Coronary Angioplasty Using Traditional vs Transformed ECG-Based Indexes. Comput Biomed Res 1999; 32:470-82.

2. Vetter R, Vesin J-M, Celka P, Scherrer U. Observer of the Human Cardiac Sympathetic Nerve Activity Using Noncausal Blind Source Separation. IEEE Trans Biomed Eng 1999;46(3):322-30.

3. McInerney T, Terzopoulos D. Topology Adaptive Deformable Surfaces for Medical
Image Volume Segmentation. IEEE Trans Med Imaging 1999;18(10):840-50.

4. Kyriacou S, Davatzikos C, Zinreich SJ, Bryan RN. Nonlinear Elastic Registration of Brain Images with Tumor Pathology Using a Biomechanical Model. IEEE Trans Med Imaging 1999;18(7):580-92.

5. Munteanu A, Cornelis J, Cristea P. WaveletBased Lossless Compression of Coronary Angiographic Images. IEEE Trans Med Imaging 1999;18(3):272-81.

6. Mori K, Hasegawa J, Suenaga Y, Toriwaki J. Automated Anatomical Labeling of the Bronchial Branch and Its Application to the Virtual Bronchoscopy System. IEEE Trans Med Imaging 2000;19(2):103-14.

7. Golland P, Kikinis R, Halle M, Umans C,
Grimson WE, Shenton ME, Richolt JA. AnatomyBrowser: A Novel Approach to Visualization and Integration of Medical Information.ComputAidSurg 1999;4:129-43.

Address of the author:

Prof. Dan Adam

Dept. of Biomedical Engineering

Technion - Israel Institute of Technology

Haifa 32000

Israel

E-mail:dan@biomed.technion.ac.il 